\title{
Como o Ensino de Programação de Computadores Pode Contribuir Com a Construção de Conhecimento na Educação Básica Uma Revisão Sistemática da Literatura
}

\author{
Juliana Ferri - julianaferri@uenp.edu.br - UENP - CCP/CLM \\ Selma dos Santos Rosa - selmasantos@ufpr.br - UENP - CCP; UFPR
}

Resumo: Neste artigo apresentamos uma Revisão Sistemática da Literatura com o objetivo de identificar pesquisas que apresentem um ou mais itens de interesse relacionados à seguinte problematização: Como o ensino de programação de computadores pode contribuir com a construção de conhecimento na Educação Básica? A revisão nos deu subsídios para aferir quais plataformas de ensino de computação e quais estratégias didático-pedagógicas estão sendo utilizadas, bem como, quais evidências de fragilidade e de potencialidade há neste contexto. Com os dados coletados, foi efetuada uma metassíntese que nos conduziu a aferição de que o ensino de programação na Educação Básica por meio de Micromundos, como por exemplo, o SuperLogo e o Scratch, pode contribuir significativamente para a construção de conhecimento e potencializar aprendizagens e resultados frente à educação atual e futura além das manifestações e exigências da sociedade.

Palavras-chave: Programação de Computadores, Educação Básica, Micromundos, SuperLogo, Scratch.

\section{How Computer Programming Teaching Can Contribute to Building Knowledge in Basic Education A Systematic Review of Literature}

\begin{abstract}
In this article we present a Systematic Review of Literature with the objective of identifying researches that present one or more items of interest related to the following problematization: How can the teaching of computer programming contribute to the construction of knowledge in Basic Education? The review gave us some insights to assess which computer teaching platforms and what pedagogical-pedagogical strategies are being used, as well as the evidence of weakness and potential in this context. With the data collected, a metaassynthesis was carried out, which led us to verify that the teaching of programming in Basic Education through Microworlds, such as SuperLogo and Scratch, can contribute significantly to the construction of knowledge and to enhance learning and learning. Results to current and future education beyond the manifestations and demands of society.
\end{abstract}

Keywords: Computer Programming, Basic Education, Microworlds, SuperLogo, Scratch.

\section{Introdução}

A atual realidade apresentada no cenário da educação, desperta o interesse de estudiosos do mundo todo para que busquem melhorias no processo de ensino e aprendizagem de crianças e adolescentes na Educação Básica. Diversos países estão buscando o acesso ao conhecimento com o uso de Tecnologias Digitais. Dentre os progressos resultantes da inclusão do ensino dessas tecnologias no currículo escolar, destaca-se a área da Ciência da Computação na Educação em Israel (GAL-EZER, 1999), no Canadá (MEO, 2008) e nos Estados Unidos, onde mais especificamente o 
modelo de currículo chama atenção para conteúdos que permitem o alcance da chamada Educação Imperativa, na qual se considera que mais importante do que aprender temas ligados às tecnologias, está a capacidade de desenvolver o pensamento computacional (CSTA, 2005).

Atualmente, o ensino de programação na educação básica constitui-se um objeto de estudo de pesquisadores das diferentes partes do mundo (MARQUES, 2009; CALDER, 2010; PINTO, 2010; CORREIA, 2012; MORALLES, 2013). Com a proposição de melhorar o raciocínio lógico dos estudantes e aumentar a criatividade na busca de soluções de problemas reais, a Inglaterra modificou o Currículo da Educação básica nacional e, em setembro de 2014 as escolas começaram a lecionar computação para as crianças das escolas primárias e secundárias (Berry, 2013). No Japão o ensino de programação já está inserido na disciplina de Matemática, da Educação Básica. Alunos das séries iniciais já começam suas primeiras atividades, enquanto desenvolvedores de programação para computador.

O tema Ferramentas Computacionais na Educação é promissor e vem sendo claramente explorado (MARQUES, 2009; CALDER, 2010; PINTO, 2010; CORREIA, 2012; MORALLES, 2013). Apesar de se tratar de uma vasta área de pesquisa com potencialidades em produções científicas, nota-se que a sociedade moderna está definida e estruturada pela tecnologia, mas o sistema escolar ainda se encontra tradicionalmente conservador, isto é, isentando essa influência. (CHANG, 2015).

Neste contexto, a discussão sobre produzir um currículo contendo conteúdos da Ciência da Computação na Educação Básica reflete um avanço no sistema educacional, já que assim, se estabeleceria um espaço para o desenvolvimento de novas habilidades, que serão demandas para os bons profissionais em um futuro muito próximo, a exemplo da capacidade de resolver problemas, de simplificar e interpretar, de liderar, trabalhar em equipe e da fluência no uso de tecnologias para quaisquer áreas do conhecimento (SCAICO, 2012).

Assim como os marceneiros não podem construir mobílias sem um conjunto de ferramentas adequado, os alunos não podem construir significado se não tiverem acesso a um conjunto de ferramentas intelectuais que os ajudem a reunir e construir conhecimento e melhorar sua estrutura cognitiva (JONASSEN, 2007). Neste sentido, os computadores podem ser utilizados como "ferramentas cognitivas", conceito definido por Jonassen (2007), cuja proposição é enfatizar o uso de tecnologias computacionais como recursos de apoio a construção de conhecimentos e ao mesmo tempo fomentar o pensamento crítico, criativo e reflexivo, considerados competências fundamentais para a Educação atual e futura (UNESCO, 2009).

São várias as ferramentas computacionais consideradas cognitivas. Entretanto, neste artigo, destacaremos os Micromundos, uma ferramenta cognitiva apresentada por Papert (1993) e por Jonassen (2007), que segue uma perspectiva construtivista da aprendizagem. Atualmente existem vários exemplos de Micromundos. Dentre eles podemos citar: (a) os que enfatizam a programação de computadores (scratch, kodu, LOGO, Superlogo e etc.) e (b) os que enfatizam a aprendizagem por meio de construção de cenários, cidades, temas relacionados à cultura, a administração, a políticas e ao meio ambiente (exemplo: minecraft).

Assim, com a proposição de dar um contributo para o estado da arte, apresentamos os resultados de uma Revisão Sistemática da Literatura (RSL), que teve como objetivo identificar pesquisas que apresentam um ou mais itens de interesse relacionados à seguinte problematização: como o ensino de programação de computadores pode contribuir com a construção de conhecimento na Educação Básica? 
A RSL aqui preconizada visa identificar quais ferramentas (cognitivas) estão sendo aplicadas no ensino de programação de computadores na Educação Básica, quais estratégias didático-pedagógicas foram desenvolvidas para validar o processo de ensino e aprendizagem dos alunos após aplicação da programação de computadores e quais os resultados atingidos.

Além dos resultados obtidos ao final da revisão, este artigo inclui o detalhamento das atividades intermediárias realizadas divididas em seções, sendo elas: o procedimento metodológico, o planejamento do protocolo da revisão sistemática da literatura, a estratégia adotada para selecionar e excluir conteúdos, utilização de mecanismos de busca automática, seleção de trabalhos, resultados e discussões estruturados por categorias, considerações finais e referências.

\section{Procedimento Metodológico}

O procedimento metodológico para este trabalho foi estruturado por meio da coleta de dados realizada com o método de investigação científica denominado Revisão Sistemática da Literatura (RSL). Com ela, objetiva-se apresentar uma avaliação a respeito de um tópico de pesquisa, fazendo uso de um método de revisão que seja confiável, rigoroso e que permita auditagem (KITCHENHAM, 2004).

O processo de RSL envolve três etapas: planejamento, condução, e apresentação dos resultados. Durante o planejamento é criado o protocolo que contém entre outros itens uma ou mais questões de pesquisa a serem respondidas, bem como critérios e estratégias para a busca e seleção de estudos relevantes (importantes para responder as questões de pesquisa). Durante a etapa de condução, são realizadas três atividades: a busca por estudos candidatos, a seleção dos estudos relevantes e a extração de dados. Por fim, durante a última etapa do processo, os resultados são sintetizados e sumarizados (KITCHENHAM, 2004).

Por conseguinte, realizamos uma análise dos trabalhos selecionados durante a RSL, por meio do método Metassíntese. Neste método, conforme Lopes et. al. (2008a) os resultados obtidos na RSL são analisados e compilados de forma qualitativa, buscando obter conclusões macro do conjunto de estudo.

\subsection{Planejamento e Condução da RSL}

O principal objetivo desta RSL foi identificar trabalhos que abordam o ensino de programação na Educação Básica, por conseguinte, identificar quais as plataformas de ensino de computação foram utilizadas, quais estratégias didático-pedagógicas foram desenvolvidas para validar o processo de ensino e aprendizagem dos alunos após aplicação da programação de computadores e quais os resultados atingidos.

\subsection{Definições do Protocolo da Revisão Sistemática da Literatura}

O planejamento da RSL foi realizado de acordo com o modelo de protocolo apresentado por Kitchenham (2004), orientado sobre uma estratégia de busca definida que visa detectar o máximo de literatura relevante possível, estratégia de busca documentada para que os leitores possam avaliar o rigor e a completude, critérios de inclusão e exclusão explícitos para avaliar cada estudo, especificar a informação a ser obtida de cada estudo, que por sua vez é pré-requisito para a meta-análise quantitativa. Nessa seção, são apresentados os principais pontos do plano elaborado.

\subsection{Objetivos Específicos da Pesquisa}

Neste artigo objetivamos: Identificar práticas de ensino de programação de computadores no processo de ensino e aprendizagem na Educação Básica; Identificar 
em quais disciplinas e séries está ocorrendo a inserção da programação de computadores; Aferir como está ocorrendo a validação da transformação do processo de ensino e aprendizagem no aluno mediante aplicação da programação de computadores.

\subsection{Questões de Pesquisa}

A fim de direcionar a pesquisa e avaliar os materiais relevantes ao tema propomos as seguintes questões de pesquisa: Q1: Como o ensino de programação de computadores está sendo inserido no processo de ensino e aprendizagem na Educação Básica? Q2: Quais estratégias e ferramentas didático-pedagógicas relacionadas ao ensino de programação de computadores, estão apoiando o processo de ensino e aprendizagem na Educação Básica? Q3: Em quais disciplinas e séries estão sendo utilizadas as plataformas de ensino de computação, na Educação Básica? Q4: Quais os benefícios no processo de ensino e aprendizagem com o uso das plataformas de ensino de computação, na Educação Básica? Q5: Quais os pontos de melhoria relacionados ao processo de inserção das plataformas de ensino de computação na Educação Básica podem ser evidenciados?

Essas questões de pesquisas podem ser estruturadas em quatro itens, identificados como PICO - Population (População), Intervation (Intervenção), Comparison (Comparação), Outcome (Resultados). Definidos com a seguinte estrutura: P: Identificar estudos primários de ensino de programação na Educação Básica; I: Verificar se está sendo aplicado o ensino de programação de computadores na Educação Básica; C: Aferir as pesquisas que possuem maior relevância de acordo com os estudos apresentados. O: Identificar os mecanismos e estratégias adotados nos estudos primários encontrados e realizar uma sumarização dos resultados.

\subsection{Estratégias de Busca Para Seleção de Estudos}

Para efeitos da revisão sistemática da literaura, foram incluídos artigos disponibilizados nas bases de dados IEEE Institute of Electrical and Electronic Engineers, ScienceDirect, ACM Digital Library, Google Acadêmico, e anais do Simpósio Brasileiro de Informática na Educação (SBIE). As bases internacionais são referências mundiais em pesquisas relacionadas às TIC, e a última - o SBIE - é um evento anual promovido pela Comissão Especial de Informática na Educação (CEIE). Esta base tem como objetivos divulgar a produção científica nacional nesta área e proporcionar um ambiente para a troca de experiências e ideias com empresários, profissionais, professores, estudantes e pesquisadores nacionais e estrangeiros.

Para a busca foram definidas as seguintes palavras-chave (em inglês e em português) inicialmente condutoras da pesquisa: $<<$ Logo Language $>>$; $<<$ Linguagem LOGO $>>$; $<<$ Programing in education basic - Programação na Educação Básica $>>$; $<<$ The programming teaching in basic education - $\mathrm{O}$ ensino de programação na Educação Básica $>>$; $<<$ Tools use in programming teaching in basic education Ferramentas usadas no ensino de programação na Educação Básica $>>$ complementadas com nomes dos principais micromundo $<<$ Scratch $>>$ e $<<$ SuperLogo $>>$ por ser uma extensão da linguagem LOGO como o MultiLogo e o PeopleLogo.

Contudo, em uma primeira busca, as palavras-chave foram associando programação, ferramentas de programação, ensino e Educação Básica no idioma português e em Inglês retornando uma quantidade de artigos muito elevada, n=1630368. Algumas bases de busca não efetuaram a concatenação esperada das palavras chave, desta forma retornaram resultados inválidos a esta RSL. Assim foi necessário efetuar uma seleção das palavras chaves inicialmente utilizadas e criar novas strings de busca com o objetivo de responder às questões de pesquisa: Programing in Education Basic; 
Programação na Educação Básica; "The programming teaching in Basic Education"; "O ensino de programação na Educação Básica"; "Tools use in programming teaching in Basic Education"; "Ferramentas usadas no ensino de programação na Educação Básica".

Nesta etapa de busca automática foram obtidos 2544 sendo: IEEE $=113$, ScienceDirect $=1240, \mathrm{ACM}=1163, \mathrm{SBIE}=4$, e Google Acadêmico $=24$, apenas 28 no idioma em português, e 2516 no idioma Inglês. Foram coletados artigos publicados entre o ano de 2000 e 2016, fomentando informações recentes sobre a problemática.

Contudo, este método de investigação científica efetuado por busca automática teve a necessidade de complemento com outra técnica de busca manual a fim de se afirmar o que foi revelado anteriormente. Desta forma utilizou-se a técnica metodológica conhecida como snowball, também divulgada como snowball sampling ("Bola de Neve").

Essa técnica é uma forma de amostra não probabilística utilizada em pesquisas sociais onde os participantes iniciais de um estudo indicam novos participantes que por sua vez indicam novos participantes e assim sucessivamente, até que seja alcançado o objetivo proposto (o "ponto de saturação") (W.H.A., 1994).

Em síntese, esta busca manual é realizada nas referências dos artigos selecionados coletando os autores mais citados por eles. A coleta de dados foi realizada entre os meses de Abril de 2016 e Agosto de 2016.

\subsection{Critérios de inclusão e exclusão}

Ao todo, foram encontrados $\mathrm{n}=2516$ artigos, que foram submetidos para as etapas de seleção preliminar, seleção final e extração de resultados. A seleção preliminar foi efetuada por meio da leitura dos títulos e palavra chave dos 2516 , sendo possível excluir 2282, estes não possuíam relevância para a pesquisa aqui preconizada que findou 234 do montante inicial para a etapa de seleção final. Efetuamos a leitura dos títulos, palavras chaves e abstracts dos 234 artigos restantes.

Após a leitura dos itens propostos, foram excluídos 210 artigos, apenas 24 foram utilizados para extração dos resultados. Os principais motivos das exclusões foram: artigos relacionados ao ensino de alunos com necessidades especiais; com foco em Educação a Distância; estudos que apresentavam algoritmos específicos para o ensino de programação; artigos com resultados insuficientes para análise; relacionados ao tratamento da dislexia com programação de robôs; materiais selecionados com a busca pela palavra chave correta, contudo, não pretextavam o contexto esperado; pesquisa direcionada ao ensino superior; formação de educadores e; software de análise de patentes relacionadas à saúde.

Efetuamos a leitura na íntegra dos 24 artigos, tendo em vista que em seus abstracts, títulos e palavras chave não apresentaram todas as informações necessárias para findar a pesquisa e apenas 7 foram selecionados. Nestes 7 aplicamos a busca em suas referências, técnica conhecida como snowball para reforçar a pesquisa, o que retornou os seguintes artigos: Contreras (2014); Sanjanaashree (2014); Burke (2012); Meerbaum-Salant (2013); Resnick (2007). O levantamento final foi concluído com 12 artigos que realmente contribuem com o objetivo da pesquisa.

Dyba e Dingsoyr (2008) apresentam três pontos principais a serem considerados na elaboração dos critérios de inclusão ou exclusão e facilitar o processo de revisão sistemática: o rigor científico, a credibilidade e a relevância. Nesta pesquisa elencamos os seguintes critérios: A programação de computadores é apresentada de maneira clara e objetiva? O contexto foi descrito adequadamente podendo contribuir para a pesquisa? 
São apresentadas outras ferramentas que podem contribuir no processo de ensino e aprendizagem aliada com o pensamento computacional? Corrobora com a Educação Básica?

\subsection{Estratégias de Extração e Sumarização dos Dados Coletados}

Os dados foram analisados e subdivididos por categorias estruturadas com o propósito de responder as questões de pesquisa e atingir o objetivo geral da RSL. Por conseguinte, por meio do método Metassíntese, o qual analisa os resultados de forma qualitativa, efetuamos a sumarização e a compilação dos dados coletados.

\section{Resultados e Discussões}

Categoria 1 - Plataformas de ensino de computação na Educação Básica - nesta categoria foi possível elencar entre os 12 artigos a seguinte relação de plataformas computacionais que são do tipo "Micromundos": Scratch $(n=7)$; SuperLogo $(n=3)$; Flash CS3 Professional ( $\mathrm{n}=1)$; e AlgoBlock ( $\mathrm{n}=1)$.

As mais citadas foram Scratch e SuperLogo, a primeira desenvolvida pelo Instituto Tecnológico de Massachussets (MIT, experiente no desenvolvimento de ferramentas educativas para crianças na idade escolar) e pelo grupo KIDS da Universidade de Califórnia, Los Angeles (SCRATCH.MIT, 2016). Trata-se de uma plataforma que inclui uma linguagem de programação visual projetada para crianças com mais de seis anos de idade, um ambiente de desenvolvimento e um site onde a comunidade pode hospedar seus projetos, executar, estudar e reutilizar outros programas além de compartilhar ideias ou sugestões com outros programadores. Nesta plataforma, são desenvolvidos e compartilhados exemplos de aplicações. Podemos notar que, esta plataforma ajuda seus usuários a pensar de forma criativa, a raciocinar sistematicamente e a trabalhar colaborativamente - competências essenciais à vida no século XXI, de acordo com a UNESCO (2009).

A segunda plataforma de maior relevância citada nos artigos pesquisados foi a linguagem LOGO desenvolvida nos Estados Unidos no Massachusetts Institute of Tecnology (MIT), na década de 60, a partir de pesquisas feitas pelos matemáticos Seymour Papert e Wallace Feurzeig, diretor da MIT. Conta-se que a ideia surgiu durante um jantar em que a proposta era a criação de uma linguagem de programação para substituir o Basic e assim nasceu a LOGO: uma linguagem com a capacidade de processar listas e de permitir a criação de procedimentos. Nessa época, a Logo não possuía uma interface gráfica, pois os computadores não tinham essa habilidade, tornando-se impossível sua implementação. Por ter sido considerada uma ferramenta de difícil entendimento para as crianças, esta linguagem de programação não foi totalmente aceita (LOPES et al 2013b). Entretanto, em 2000, o Núcleo de Informática Educativa à Educação (NIED) da Universidade de Campinas tendo como base a proposta da LOGO, criou a SuperLogo. Existem várias versões da LOGO, contudo nesta investigação, encontramos nos artigos a utilização da versão SuperLogo $3.0(n=3)$, que é a linguagem de programação original adaptada para o português pelo NIED.

Categoria 2 - Estratégias didático-pedagógicas - em suma, as estratégias didáticopedagógicas aferidas durante a RSL focam-se na resolução de problemas, e na resolução de desafios, com vistas a trabalhar a criatividade dos alunos e suas habilidades cognitivas. Alguns estudos foram realizados em grupos pequenos e aos pares As estratégias eram aplicadas e validadas em oficinas e projetos em atividades extracurriculares e em contra turno por períodos pequenos. Ao término deste período, eram realizados relatos por meio de relatórios que continham o estudo de caso da estratégia adotada. 
Souza, 2015, utilizou fóruns de discussão sobre a SuperLogo, e a construção de materiais produzidos pelos participantes do projeto ao longo do processo de planejamento de aulas que propunham o uso dessa plataforma.

Os autores, Ramos (2015), Molina-García (2016), Sapounidis (2015), Nicholas (2012), Meerbaum-Salant (2010) desenvolveram projetos com vistas ao uso de plataformas computacionais aplicados a grupos formados por meninas e meninos e atividades em pares. Os artigos apresentaram um mínimo de 13 e máximo de 121 alunos divididos em grupos menores e maiores. Nos projetos apresentados os alunos desenvolviam inicialmente atividades propostas seguindo uma sequência pré-definida e outra para usar a criatividade. Além de atividades em grupos também desenvolveram atividades em pares.

Identificamos 2 artigos, Contreras (2014) e Souza (2015) que desenvolveram estratégias com o uso das plataformas computacionais por meio de estudos de caso com professores da Educação Básica e professores do ensino de design de diferentes países sendo o foco do estudo, encarregados de aulas relacionadas com o ensino de computador ou encarregados de desenvolver o currículo da escola.

Categoria 3 - Aplicação das estratégias correlatas às áreas do conhecimento - Dos artigos pesquisados $(\mathrm{n}=12), 4$ aplicaram as plataformas computacionais associadas ao ensino de matemática e geometria (ROCHA, 2015; MOTTA, 2010; SOUZA, 2015; NICHOLAS, 2012); 5 estavam relacionados ao ensino de programação de computadores, (MOLINA-GARCÍA, 2016; CONTRERAS, 2014; MEERBAUMSALANT, 2010; SAPOUNIDIS, 2015; RESNICK, 2007); 1 artigo apresenta um projeto entre alunos e professores com a perspectiva de permitir a mudança de comportamento e promover formas inovadoras de ensino e de produção de materiais em diversas áreas do conhecimento (RAMOS, 2015); 1 aplica o Scratch para o ensino de uma língua secundária, baseando-se no conceito de aprendizagem visual e auditiva. Segundo Sanjanaashree (2014), quando a aprendizagem é por meio de uma ferramenta de visualização e auditiva, traz a compreensão em profundidade dos dados e a sua sequência de fonemas através de animação e pronúncia correta das palavras, o que é muito melhor do que as pessoas aprendem a partir dos livros e imaginando em sua perspectiva e com sua própria pronúncia.

O último artigo relatado utilizou a plataforma computacional Scratch para criação de histórias digitais contadas pelos próprios alunos. Nesta investigação, verificou-se o potencial de introduzir conceitos básicos de programação para as crianças do ensino médio dentro do contexto de escrita e de leitura (BURKE, 2012).

Categoria 4 - Evidências de fragilidade - Uma possível evidência de fragilidade foi constatada ao observar que de todos os artigos selecionados, nenhum apresentou o uso de plataformas computacionais no currículo básico escolar, somente de maneira transversal ao currículo por meio de estudos de caso realizados em oficinas com pequenas populações, projetos de contra turno, projetos de verão, ou estudo bibliográfico sobre o assunto.

Há pouca pesquisa que explora sistematicamente as vantagens cognitivas e sociais do uso das plataformas computacionais bem como o uso das interfaces gráficas, em particular, o impacto em ambientes reais e as condições em que a manipulação de objetos e materiais pode ser mais eficiente para crianças, em vários domínios, tais como a programação, não têm sido estudados o suficiente e permanecem essencialmente inexplorado (SAPOUNIDIS, 2015).

A proposta de utilizar linguagens de programação para desenvolver habilidades dos alunos não é muito recente. O desenvolvimento da LOGO para fins educacionais, em 1967 (VALENTE, 1993), ilustra bem isso. No entanto, apesar de experimentado em 
diversas partes do mundo, ao longo dos anos, os resultados com a LOGO, em geral, ficaram aquém das expectativas (RESNICK et al., 2009). Por outro lado, os desenvolvedores da linguagem Scratch, conscientes desse panorama e ao mesmo tempo confiantes na proposta educacional do uso de linguagens de programação, estabeleceram três princípios centrais de design para ela: torná-la mais acessível, mais significativa e mais social do que as propostas anteriores (RESNICK et al., 2009).

Categoria 5 - Evidência de potencialidade - Foi possível aferir nos artigos selecionados $(n=12)$, que os participantes dos projetos ou estudos de caso propostos com a inserção do pensamento computacional, é capaz de despertar a atenção dos alunos. Durante o estudo notas de observação foram tomadas pelos professores constatando que os envolvidos foram geralmente atenciosos durante todo o processo de desenvolvimento das tarefas propostas (NICHOLAS, 2012).

Foi observado que com a inserção da cultura tecnológica no processo de ensino e aprendizagem dos alunos é possível despertar capacidades lógicas e incorporá-las na solução de problemas do seu cotidiano. Ensinar programação para alunos de níveis básicos tem o objetivo de prepará-los para um mercado de trabalho mais produtivo, e reforçar o seu conhecimento acadêmico em diferentes disciplinas (MOLINA-GARCÍA, 2016). É possível criar estratégias de inserção do pensamento computacional a fim de melhorar o desempenho dos alunos já na Educação Básica.

\section{Considerações finais}

Podemos citar várias áreas de melhoria observadas nos artigos selecionados tais como o comportamento dentro e fora da sala de aula, as relações interpessoais construídas entre os grupos, a organização de ideias e competências tecnológicas, desenvolvimento de capacidades de compreensão e expressão oral e escrita, além de favorecer a concentração e motivação dos alunos.

Na perspectiva da utilização dos Micromundos, o aluno traz a teoria dos livros, das salas de aulas para dentro do computador e aplica os conceitos anteriormente aprendidos em um mundo virtual, criando cenários, animações, despertando o senso crítico e ultrapassando desafios que futuramente podem acontecer na vida real, desta forma já estarão preparados para os desafios da sociedade atual e futura.

Contudo, apesar de todas as vantagens educacionais supracitadas sobre o uso da linguagem de programação no Brasil, ainda há poucas pesquisas que divulgam projetos nesta área, desenvolvidos na Educação Básica, tendo em vista que esta é uma questão imensurável à medida que nem todas as experiências educacionais necessariamente são registradas em artigos acadêmicos.

\section{Referências}

BERRY, M. Computing in the national curriculum: a guide for primary teacher. Newnorth Print, Ltd. Bedford. Acesso em: http://www.computingatschool.org.uk/data/uploads/CASPrimaryComputing.pdf BURKE, Q.; KAFAI; Y. B. The Writers Workshop for Youth Programmers Digital Storytelling with Scratch in Middle School Classrooms. pp. 433-438, 2012.

SIGCSE'12, February 29-March 3, 2012, Raleigh, North Carolina, USA. Copyright 2012 ACM 978-1-4503-1098-7/12/02.

\section{CALDER, N. "Using Scratch: An Integrated Problem-solving Approach to}

Mathematical Thinking", Australian Primary Mathematics Classroom , 15, 4, pp. 9-14 - 2010. 
CHANG, S.; DE PAULA, R.; TOSTES, A. M. B.; BARBOSA, G. S. Uma Investigação sobre o Uso de Ferramentas Computacionais no Ensino de Cálculo Integral e Diferencial. Renote - Revista Novas Tecnologias na Educação. n. 2010. v. 13, n. 2 (2015).

COMPUTER SCIENCE TEACHER ASSOCIATION - CSTA. The New Educational Imperative: Improving High School Computer Science Education. Final Report of the CSTA, 2005. Curriculum Improvement Task Force. ACM - Association for Computing Machinery.

CONTRERAS, G. J.; WAI, K.; SIU, M. Computer Programming For All : A CaseStudy In Product Design Education . Procedia - Soc. Behav. Sci., vol. 182, pp. 388 394, 2015.

CORREIA, M. M. P. dos R. K. da S. Integração dos recursos educativos digitais no 1 ciclo do ensino básico: uma realidade ou uma utopia. Tese de Metrado. Lisboa: Escola Superior de Educação João de Deus. 2012.

DYBA, T.; SJOBERG, D. I.; JORGENSEN, M. The future of empirical methods in software engineering research, in Future of Software Engineering (FOSE), pp. 358378, 2007.

Eletrônica R, Pós-graduação P De, Ambiental E, Pesquisa ATDE, Bola S. Universidade Federal do Rio Grande - FURG. 2011:46-60.

GARCIA, R. E.; CORREIA, R. C. M.; SHIMABUKURO, M. H. Ensino de Lógica de Programação e Estruturas de Dados para Alunos do Ensino Médio. Anais do XXVIII WEI, CSBC'2008, Belém.

JONASSEN, D. H. Computadores Ferramenta Cognitivas. Desenvolver o Pensamento Crítico Nas Escolas. Porto Editora. 2007.

KITCHENHAM, B. Procedures for performing systematic reviews Technical Report. TR/SE-0401, Keele University and NICTA, 2004.

LOPES, A. L. M.; FRACOLLI, L. A. Revisão sistemática de literatura e metassíntese qualitativa: considerações sobre sua aplicação na pesquisa em enfermagem. 2008. Texto contexto - enfermagem, Florianópolis, v. 17, n. 4, p. 771778. DOI: $10.1590 / \mathrm{S} 0104-07072008000400020$.

LOPES, A.; COUTINHO, C. Programar para prevenir: o uso do Scratch na segurança na internet. Anais do Challenges. Braga. Portugal. 2013.

MARQUES, M.T.P.M. Recuperar o engenho a partir da necessidade, com recurso às tecnologias educativas: contributo do ambiente gráfico de programação Scratch em contexto formal de aprendizagem. Dissertação de mestrado não publicada. Universidade de Lisboa, Faculdade de Psicologia e de Ciências da Educação, 2009.

MEERBAUM-SALANT, O; BEN-ARI, M. M. Learning Computer Science Concepts With Scratch. ICER 2010, August 9-10, 2010, Aarhus, Denmark. Copyright 2010 ACM 978-1-4503-0257-9/10/08 pp. 69-76.

MORALLES, A. Programação de computadores para melhoria da aprendizagem. Cadernos de Gestão e Empreendedorismo. V. 1, n.2. Set-dez. 2013.

MORENO, J; ROBLES, G. Automatic Detection of Bad Programming Habits in Scratch : A Preliminary Study. IEEE. 2014. 978-1-4799-3922-0/14/

MOTTA, M. S.; SILVEIRA, I. F. Contribuições do Superlogo ao Ensino de Geometria. Informática Na Educação: Teoria \& Prática. Porto Alegre, v.13, n.1, jan./jun. 2010. ISSN digital 1982-1654 ISSN impresso 1516-084X. 
MOLINA-GARCÍA, J. C.; RODRÍGUEZ-ELÍAS, M. O.; RODRÍGUEZ-PÉREZ, J. M.; GLASSERMAN-MORALES, L. D. Designing A Strategy Of Programming Learning For Kids Through The Use Of The "Micompu.Mx" Federal Program. A Pilot Study. 4th International Conference in Software Engineering Research and Innovation Designing. DOI 10.1109/CONISOFT.2016.24. 2016.

NICHOLAS, Z. The Use Of Information And Communication Technologies In The First Grade Of Primary School For Teaching Rectangles Based In Realistic Mathematics Education. Department of Preschool Education University of Crete Rethymno, Greece. 2012.

SCRATCH. Disponível em: $<$ https://scratch.mit.edu/about/ $>$. Acesso em 03 de out. de 2016.

PAPERT, Seymour. Mindstorms: Children, Computers, And Powerful Ideas. Da Capo Press. 1993.

PINTO, A. S. Scratch na aprendizagem da matemática no $10^{\circ}$ ciclo do ensino básico : estudo de caso na resolução de problemas. Dissertação de mestrado NAO PUBLICADA em Estudos da Criança. Universidade do Minho. 2010.

RAMOS, V. P. ICT In The Curriculum Using Scratch With Students Of The Alternative Curriculum. IEEE pp. 69-72. 2015.

RESNICK, M.. All I Really Need to Know ( About Creative Thinking ) I Learned ( By Studying How Children Learn ) in Kindergarten *. Presented at Creativity \& Cognition conference, June 2007 no. 1986, 2007.

ROCHA, K. C. Programando Com O Scratch Na Aula De Matemática. CINTEDUFRGS - Renote - Revista Novas Tecnologias na Educação. V.13 n2, dezembro, 2015.

SANJANAASHREE, P; KUMAR, A. M; SOMAN, K.P. Language Learning for Visual and Auditory Learners Using Scratch Toolkit. IEEE. 2014. pp. 1-5, 2014. 978-1-47992352-6/14/.

SCAICO, P. D. ; HENRIQUE, M. S.; CUNHA, F. O. M.; ALENCAR, Y. M. Um Relato de Experiências de Estagiários da Licenciatura em Computação com o Ensino de Computação para Crianças. Renote - Revista Novas Tecnologias na Educação. V. 10 n. 3, Dezembro, 2012.

SAPOUNIDIS, T.; DEMETRIADIS, S. Evaluating Children Performance With Graphical And Tangible Robot Programming Tools. pp. 225-237, Pers Ubiquit Comput (2015) 19:225-237. 2015.

SOUZA, A. P. G.; PASSOS, C. L. B. Dialogando Sobre E Planejando Com O Superlogo No Ensino De Matemática Dos Anos Iniciais. ISSN 1980-4415 DOI: http://dx.doi.org/10.1590/1980-4415v29n53a12. Bolema, Rio Claro (SP), v. 29, n. 53, p. 1023-1042, dez. 2015.

UNESCO. Organização das Nações Unidas para a Educação, a Ciência e a Cultura.

Educação e aprendizagem para todos: olhares dos cinco continentes. - Brasília: Ministério da Educação, 2009. http://www.cbie2016.facom.ufu.br/pt/eventos/sbie.

VALENTE, J. A. Computadores e conhecimento: repensando a educação. Campinas: Gráfica da UNICAMP, [s.n], 1993.

WORLD HEALTH ASSOCIATION. Division of Mental Health. Qualitative Research for Health Programmes. Geneva:, 1994. 